\title{
REVIEWS
}

Adv Clin Exp Med 2015, 24, 4, 679-686

(C) Copyright by Wroclaw Medical University

DOI: $10.17219 /$ acem/33009

ISSN 1899-5276

\author{
Marcelo C. Goiato ${ }^{1, A}$, F, Emily Freitas ${ }^{1, B, D}$, Daniela dos Santos ${ }^{1, \text { E, }}$ \\ Rodrigo de Medeiros $^{2,}$, , Mariana Sonego ${ }^{2, B, D}$
}

\section{Acrylic Resin Cytotoxicity for Denture Base - Literature Review}

${ }^{1} 1^{\text {st }}$ Department of Dental Materials and Prosthodontics, Aracatuba Dental School, UNESP - Universidade Estadual Paulista "Júlio de Mesquita Filho", Araçatuba, Sao Paulo, Brazil

${ }^{2}$ Department of Dental Materials and Prosthodontics, Aracatuba Dental School, UNESP - Universidade Estadual Paulista "Júlio de Mesquita Filho", Araçatuba, Sao Paulo, Brazil

A - research concept and design; $\mathbf{B}$ - collection and/or assembly of data; C - data analysis and interpretation;

$\mathbf{D}$ - writing the article; $\mathbf{E}$ - critical revision of the article; $\mathbf{F}$ - final approval of article; $\mathbf{G}$ - other

\begin{abstract}
Acrylic resin is a widely used material in clinical practice, and a satisfactory biocompatibility is essential. When the resin polymerization reaction is incomplete, residual monomers are released into the oral cavity. The aim of this study was to evaluate, through a literature review, the cytotoxicity caused by the denture base acrylic resin used, and its components. The selection of published studies was performed on the Pubmed database from January 2008 to July 2013. The keywords used were: "cytotoxicity and acrylic resins", "cytotoxicity and denture base resins" and "cytotoxicity and oral prosthesis". Inclusion criteria were: in vitro studies and literature reviews published in English that evaluated the acrylic resin cytotoxicity for denture base and its components. Studies with no reference to the search strategy were excluded. A total of 182 articles were found. Among these, only 13 were included for writing this review. The MTT test is the most common test used to evaluate acrylic resin cytotoxicity. Auto-polymerized resin is more cytotoxic than heat-polymerized resin because of its higher quantity of residual monomers which cause cell and tissue changes in the oral mucosa. However, more studies are necessary for the development of biocompatible materials (Adv Clin Exp Med 2015, 24, 4, 679-686).
\end{abstract}

Key words: acrylic resins, cytotoxicity tests, biocompatibility testing.

Acrylic resin (PMMA - Polymethylmethacrylate) is a widely used material in dentistry [1-4]. Due to its utilization in temporary crowns, denture fabrication, reline and repair, orthodontic appliances, splints for orthognathic surgery and others, this material should have the appropriate physical, chemical and biological properties [2, 3, 5-7].

Acrylic resin polymerization is crucial for the optimization of the material's physical and biological properties because it allows the conversion of monomers into polymers [8]. Residual monomers and toxic chemical products such as formaldehyde, methacrylic acid, benzoic acid, dibutyl phthalate, phenyl benzoate, phenyl salicylate and, especially, MMA (methyl methacrylate), are produced on the denture base when the polymerization process is incomplete $[2,3,5,6,8-11]$. These monomers, released in the aqueous environment of the oral cavity, are present even when polymerization is performed according to the manufacturer's recommendations [6, 9-11].

The cytotoxicity of acrylic resins and their components is evaluated by in vitro studies [1-3, $5-8,10-13]$. The cytotoxicity test is performed with extracts of acrylic resin specimens, which indirectly measure the material's biocompatibility, by their action on cell cultures $[2,6,9,14]$.

This test can also determine the toxic concentrations of tested material and its consequences on cell morphology and growth, degree of cellular damage and enzymatic activities in a particular cell type. In other words, this test defines the material's biological behavior and its components $[2,6,9]$.

The methods for cytotoxicity analysis are described and regulated by ISO-standard 10993-5 $[1,8,11]$. Bural et al. [8] reported that this classi- 
fication is based on the cytotoxicity degree of the tested material as follows: non-cytotoxicity (cell proliferation greater than $75 \%$ ), slight cytotoxicity (50-75\% of cell proliferation), moderate cytotoxicity (25-50\% of cell proliferation) and high cytotoxicity (cell proliferation less than 25\%).

The purpose of this study was to (1) review the published studies regarding the cytotoxicity of acrylic resin for denture bases and its components, (2) identify tests used for cytotoxicity analysis and (3) different responses according to the acrylic resin polymerization methods.

\section{Material and Methods}

The published studies selection was performed on the Pubmed database from January 2008 to July 2013. The keywords used were: "cytotoxicity and acrylic resins", "cytotoxicity and denture base resins" and "cytotoxicity and oral prosthesis". Inclusion criteria were: in vitro studies and literature reviews published in English that evaluated the acrylic resin cytotoxicity for denture bases and its components. Studies with no reference to the search strategy were excluded. Two reviewers read the selected studies and their information was analyzed and discussed for writing this review.

\section{Results and Discussion}

A total of 182 articles were found and 19 studies were selected according to the search strategy (Table 1). Figure 1 presents the study flow chart.

\section{Cytotoxicity Tests}

Different methods are used for cytotoxicity analysis in the literature. Among them, the most common is the MTT test (3-(4,5-dimethylthiazol2-yl)-2,5-diphenyltetrazolium), which quantifies the mitochondrial succinate dehydrogenase enzyme activity and measures the conversion of water-soluble tetrazolium salt in insoluble blue formazan by spectrophotometry. This test is an excellent marker of cell survival because it evaluates cellular respiratory activity [1, 2, 5, 9, 10, 12].

The flow cytometry technique can be performed to evaluate the cell viability and apoptosis $[3,6,7]$. In addition, a cell proliferation analysis can be executed using bromodeoxyuridine incorporation (BrdU) [1] or XTT assay (sodium 3' -[1-phenylaminocarbonyl)-3,4-tetrazolium]bis(4-methoxy-6-nitro)benzenesulphonic acid), which measure the reduction of XTT in soluble formazan product [8]. The gene expression is evaluated by the RTPCR (reverse transcription-polymerase chain reaction) which analyzes the mRNA production for collagen and other proteins and enzymes $[3,4,6]$. Furthermore, a cell's DNA and RNA synthesis can be measured by ${ }^{3} \mathrm{H}$-thymidine and $\mathrm{H}$-uridine assays, respectively $[5,9,10]$.

However, these tests are rarely performed because of their high cost, advanced technology requirement and radioactive wastes [5, 10]. Enzyme-linked immunosorbent assay (ELISA) is an immunoenzyme method that allows detection of specific antibodies and is used for cytotoxicity analysis $[10,13]$. The comet assay evaluates DNA damage (genotoxicity) [1]. Cellular lipid metabolism and cell morphology analysis are other methods for cytotoxicity evaluation $[6,9]$.

The in vitro test advantages, such as the MTT test, include the facility of implementation in different cell types, reproducibility, precise control of variables and cost-effectiveness $[5,8,10]$. However, this test does not completely represent the material cytotoxic properties in their clinical condition. Therefore, such results can not be extrapolated to the general population. The reason is that the oral mucosa, due to keratin and mucin layers, is more resistant to toxic substances than cell cultures $[2,6,8,10]$.

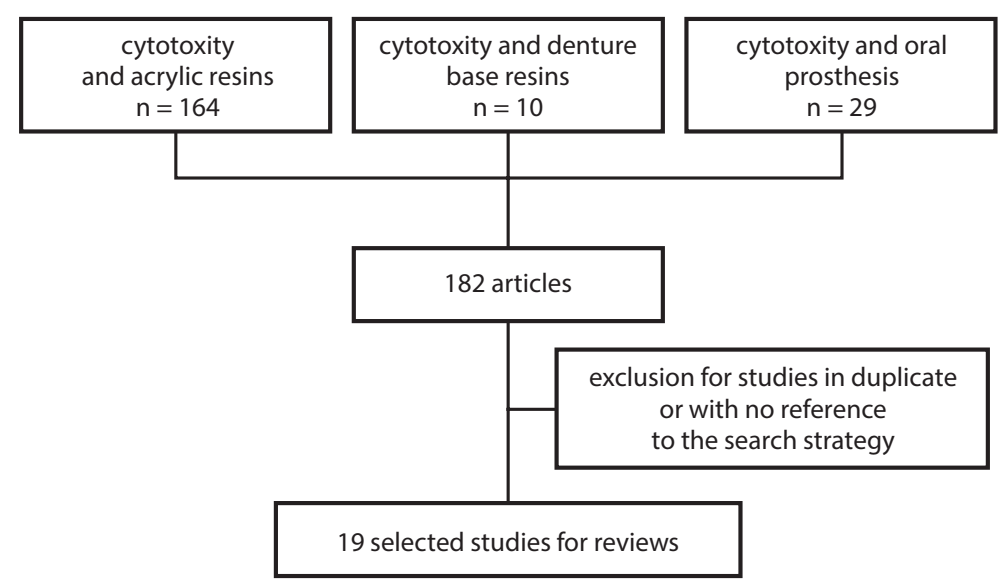

Fig. 1. Flow chart of selected publications for inclusion in the review 


\begin{tabular}{|c|c|c|c|c|c|c|}
\hline 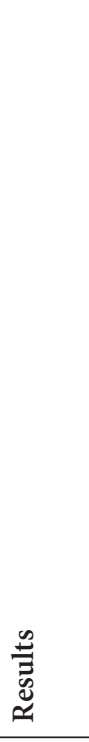 & 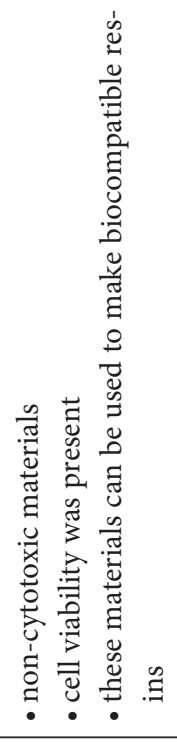 & 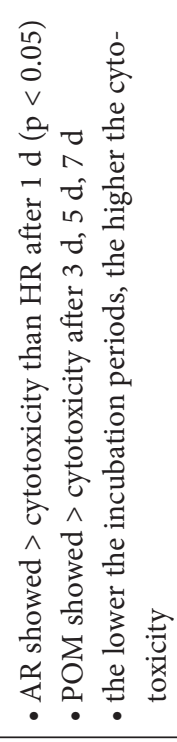 & 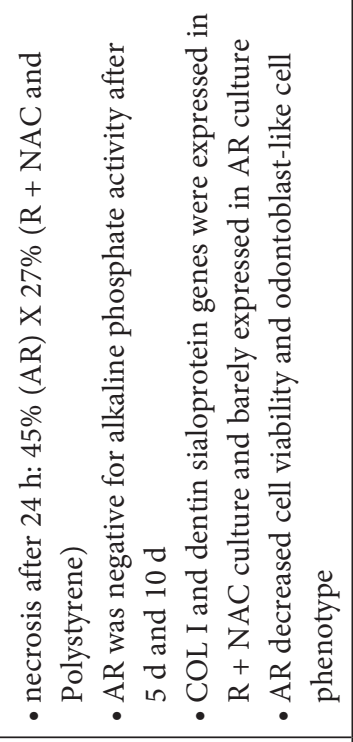 & 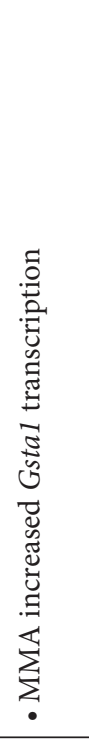 & 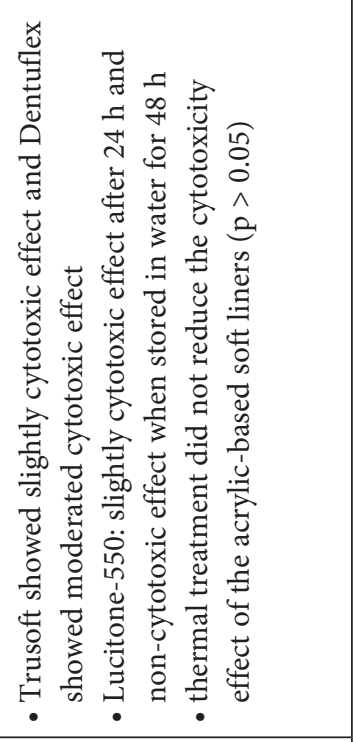 & 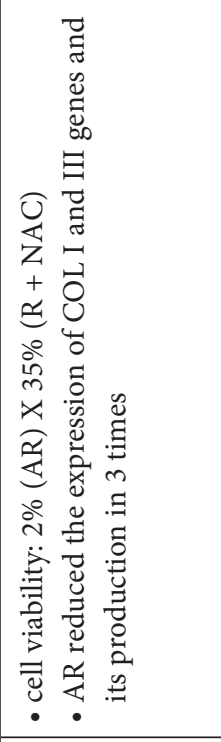 \\
\hline 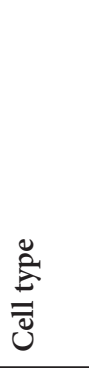 & 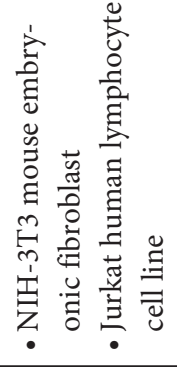 & 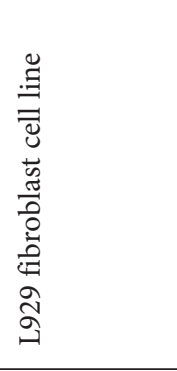 & 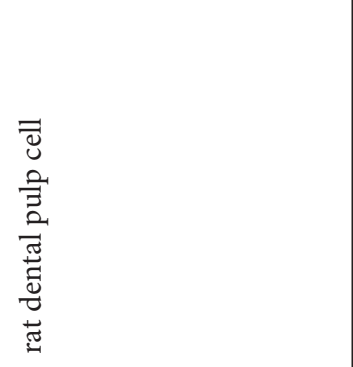 & 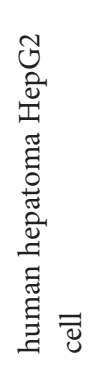 & 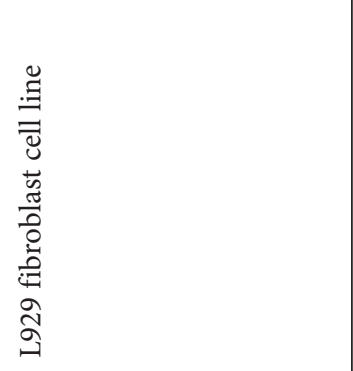 & 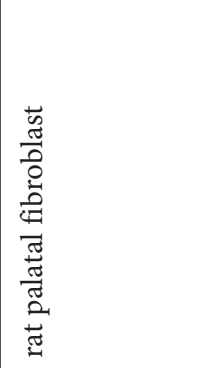 \\
\hline 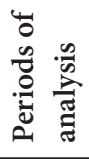 & $\begin{array}{l}\vec{A} \\
\mathbb{N} \\
\hat{E} \\
\stackrel{N}{N}\end{array}$ & 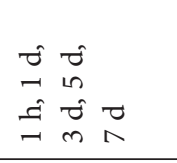 & 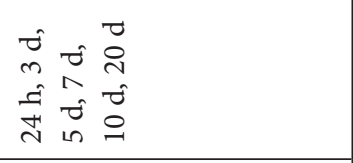 & 1 & 1 & 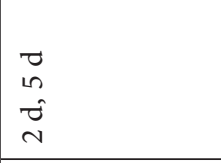 \\
\hline $\begin{array}{l}\text { 密 } \\
\frac{\operatorname{m}}{4}\end{array}$ & 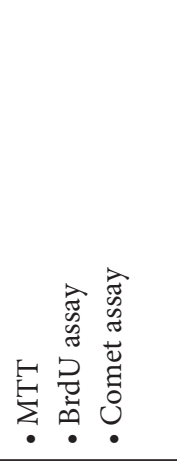 & ${ }_{\underline{\Sigma}}^{H}$ & 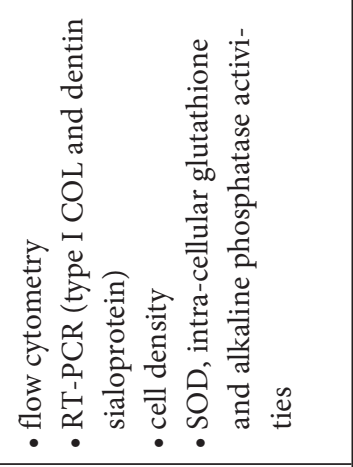 & 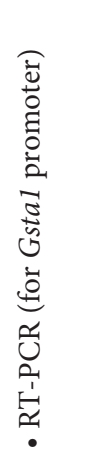 & 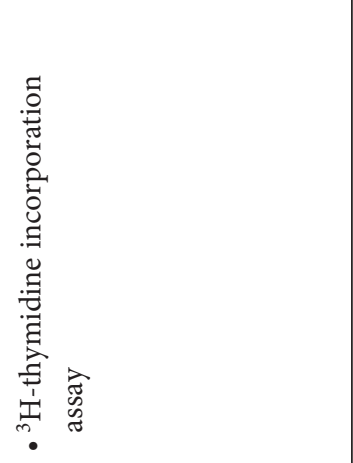 & 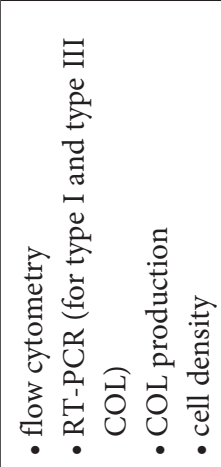 \\
\hline 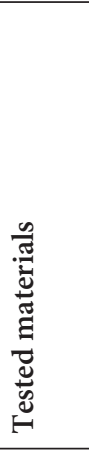 & 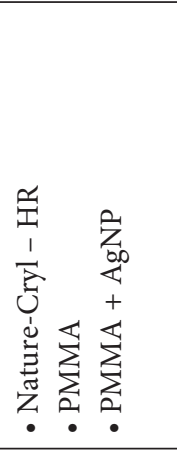 & 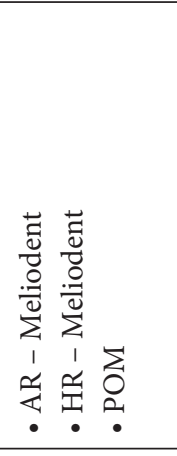 & 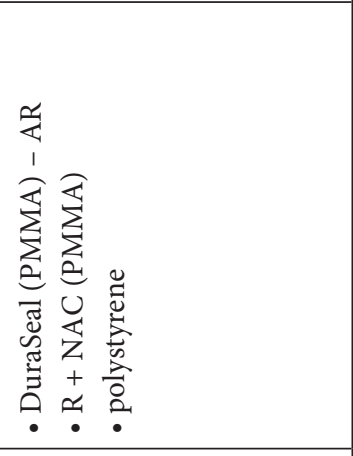 & $\sum_{\dot{\Sigma}}^{\mathbb{S}}$ & 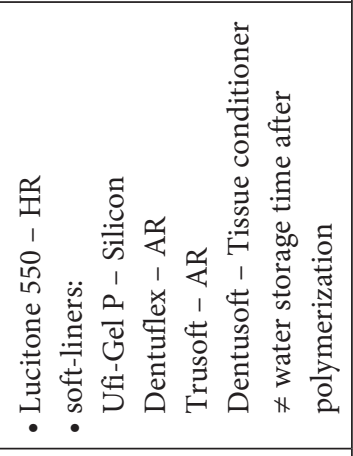 & 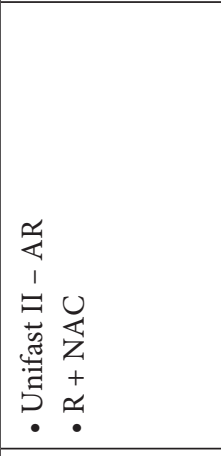 \\
\hline 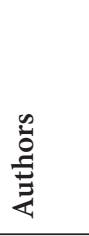 & 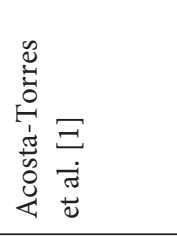 & 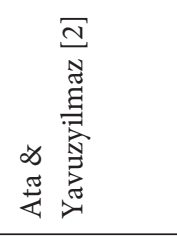 & 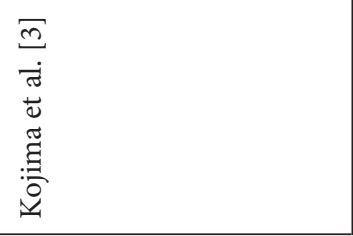 & 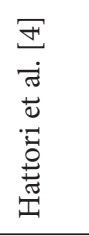 & 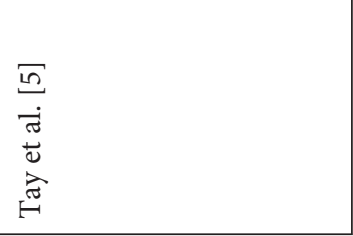 & 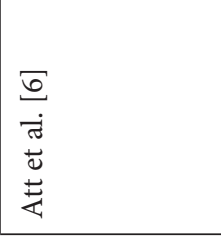 \\
\hline
\end{tabular}




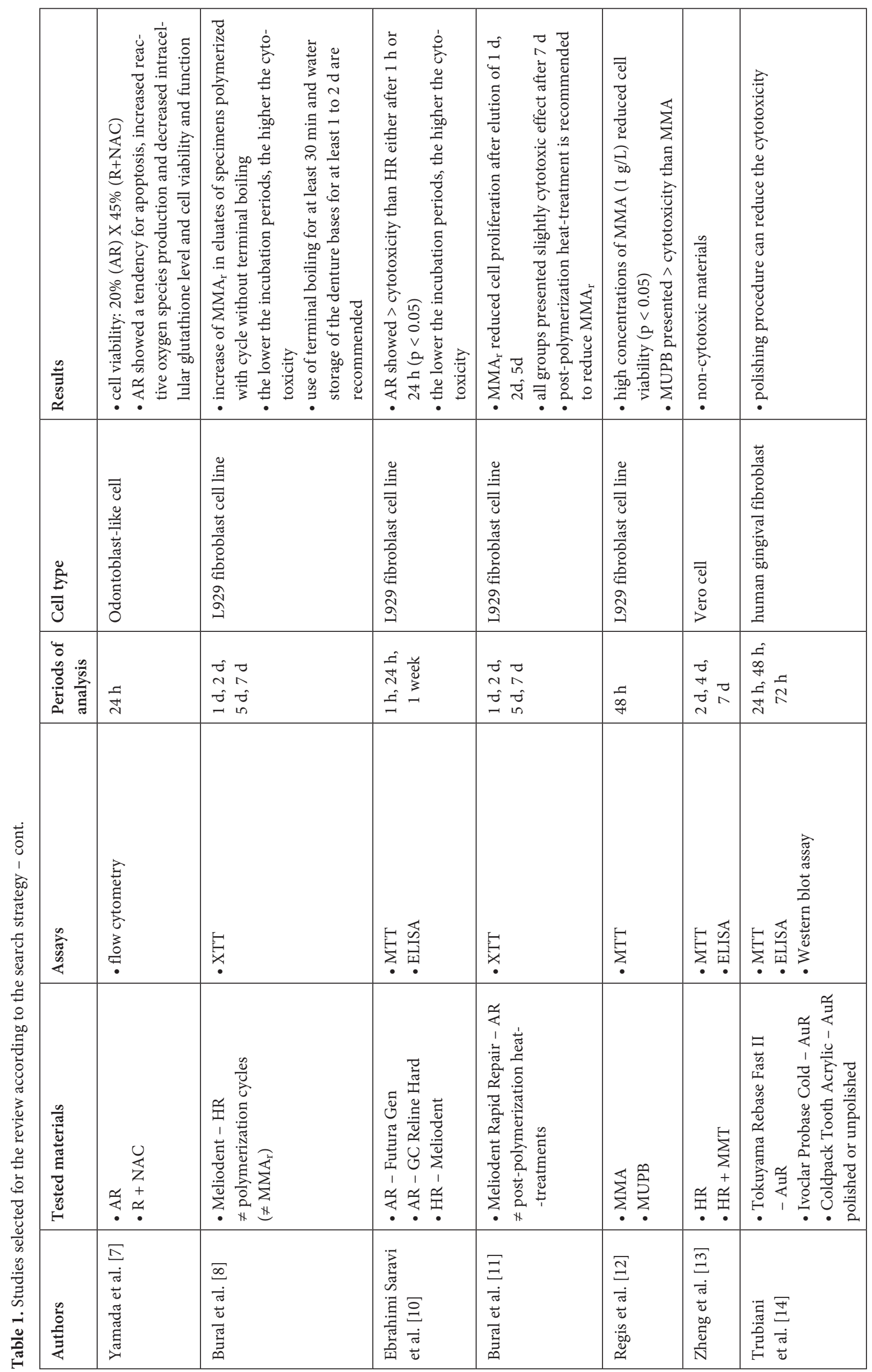




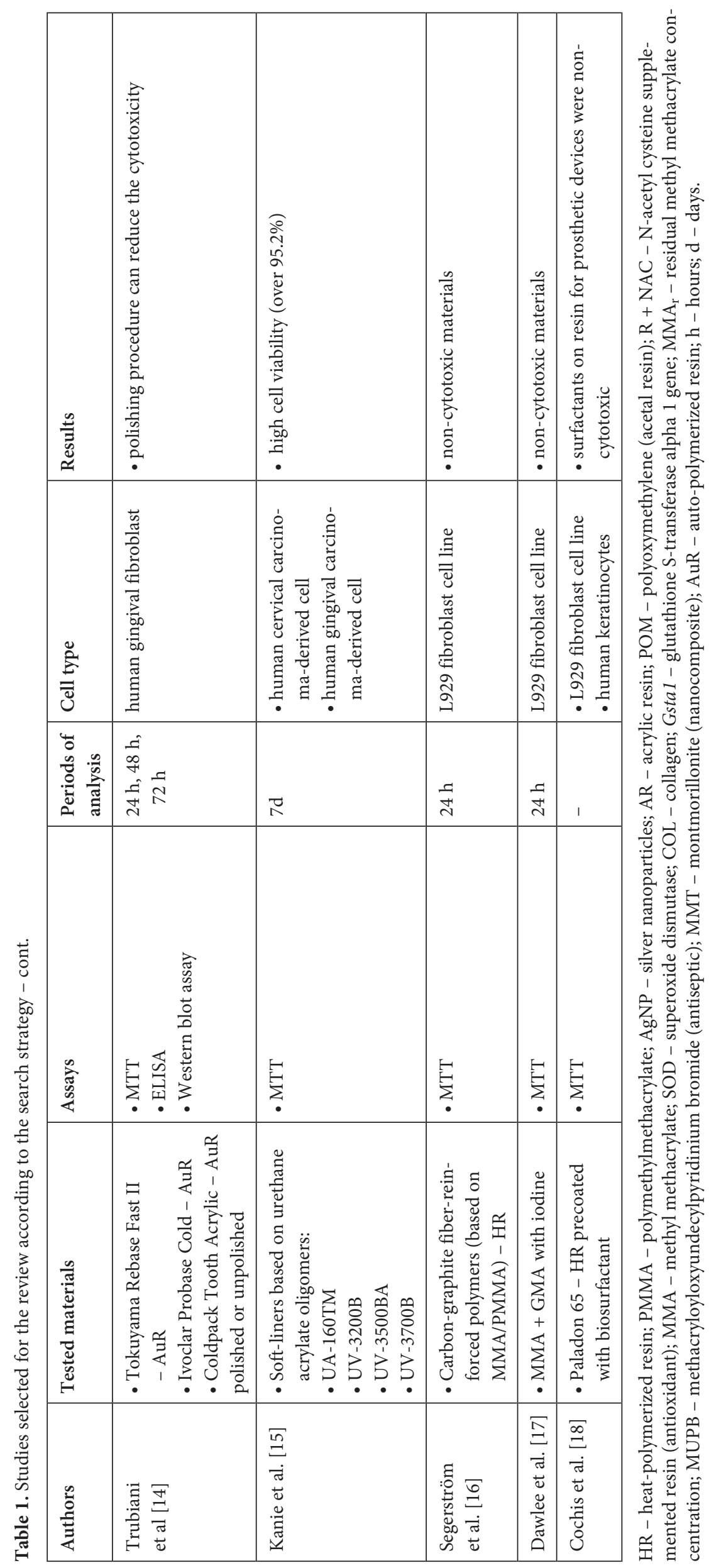




\section{Polymerization Methods}

According to Ebrahimi Saravi et al. [10], Tay et al. [5] and Ata \& Yavuzyilmaz [2], factors such as polymerization methods, temperature, the cycle of polymerization and acrylic resin storage time can influence the monomer quantity and the material cytotoxicity.

Based on the polymerization method, acrylic resin can be classified as heat-polymerized, microwave-polymerized, light-polymerized and autopolymerized, the latter being the most common in dental practice $[2,6]$. Its composition consists of a solid part of PMMA and a liquid part of MMA monomer $[3,6]$. The low cost, the use at room temperature, the short process time and no additional equipment requirement for its manipulation are some of its advantages [11]. But, heat-polymerized resin is frequently used for denture base fabrication which maintains intimate contact with oral mucosa. Similarly, its composition is based on PMMA $[8,10]$.

When different polymerization methods are compared, auto-polymerized acrylic resin releases more residual monomers than heat-polymerized resin since the increase of temperature in the latter results in molecular chain movement which converts monomers into polymers [10]. Ebrahimi Saravi et al. [10] observed that auto-polymerized resin exhibited higher cytotoxicity level than heat-polymerized resin after 1 and $24 \mathrm{~h}$ of incubation. Similarly, Ata \& Yavuzyilmaz [2] reported significant higher cytotoxicity of auto-polymerized resin in a $24 \mathrm{~h}$ period. According to Ebrahimi Saravi et al. [10], lower levels were observed after $24 \mathrm{~h}$ because of the lower monomer levels in contact with cells. So, the cytotoxicity effect is dose-dependent.

Trubiani et al. [14] compared the cytotoxic effect of three polished and unpolished auto-polymerized acrylic resins. Cell growth reduction and an increase of pro-inflammatory cytokines were caused by the tested material, specially for unpolished resins. Therefore, the polishing procedure is important in clinical practice to reduce gingival inflammation.

Post-polymerization heat treatments, such as water bath or microwave irradiation, have been suggested in order to reduce the quantity of auto-polymerized acrylic resin residual monomers $[5,10,11]$. Bural et al. [11] affirmed that groups which were submitted to water immersion showed a reduction in MMA monomer formation when compared to untreated groups. Thus, water immersion is recommended. Similarly, Bural et al. [8] evaluated different polymerization cycles of heatpolymerized resins and observed an increase of
MMA residual monomers in specimens that were not immersed in water. Therefore, the longer the prosthesis is maintained in water media prior to its installation, the greater the monomer diffusion and less damage to the patients $[5,10]$.

However, Tay et al. [5] compared the cytotoxic effect of silicon-based soft liner, acrylic-based soft liner and tissue conditioner, and found that, despite the soft liner resins having shown slight to moderate cytotoxicity levels, heat-treatment did not reduce their cytotoxic effect. Kanie et al. [15] evaluated different soft lining materials based on urethane acrylate oligomers and observed that all of them showed high cell viability.

\section{Acrylic Resin Cytotoxicity}

Biocompatibility or cytotoxicity absence is defined by the material's ability to perform its function without inducing undesirable local or systemic effects [2].

Several substances such as N-Acetyl cysteine (NAC) [3, 6, 7], silver nanoparticles (AgNP) [1] and montmorillonite nanocomposite [13] were incorporated in the acrylic resin in order to evaluate their cytotoxic effects. Carbon-graphite fiber-reinforced composites (based on MMA/PMMA) [16] and associations between MMA and glycidyl methacrylate (GMA) with elemental iodine [17] were also evaluated for cytotoxicity. The authors observed biocompatibility of the tested substances.

Regis et al. [12] compared the cytotoxicity of the MMA monomer in relation to methacryloyloxyundecylpyridinium bromide (MUPB) and identified that, despite the fact that MUPB is more cytotoxic than MMA monomer, high concentrations of MMA reduces a cell's viability.

Cochis et al. [18] evaluated the influence of a precoating with biosurfactants that prevents Candida albicans biofilm formation on the cytotoxicity of acrylic resin for heat-polymerized denture base and silicon material. The biosurfactants on prosthetic materials were non-cytotoxic. Additionally, the authors observed that the biosurfactants reduced the biofilm activity.

Although Ata \& Yavuzyilmaz [2] affirmed that acrylic resin is a low biological risk material, several authors have reported signs and symptoms in patients as a result of its residual monomer exposure. Local chemical irritation $[8,9,19]$, hypersensitivity, mucosal inflammation and ulceration $[8,9,11]$, burning sensation in the palatal mucosa, tongue, oral mucosa and oropharynx, pain, edema, swelling and local erythema and labial edema $[5,9]$ and respiratory irritation [19] are reported clinical conditions.

Additionally, fibrosis, necrosis, histiocytosis $[3,6]$, inflammatory infiltrate and a thicker 
keratin layer [5] were observed in the tissues in contact with the acrylic resin. Cytotoxicity has been reported in the literature as a result of exposure to unpolymerized resin components which cause genetic cell damage and oxidative stress $[3,6,7]$.

Hattori et al. [4] studied the effect of MMA on mRNA expression of the glutathione S-transferase alpha 1 gene (Gsta1), presented in high amounts in hepatocytes, and observed that MMA increased Gstal transcription. It is known that the liver is an organ related to detoxification. Therefore, MMA can induce cytotoxic effects in hepatocytes.

Changes in basic cell structure and function include the loss of membrane integrity, alteration of enzyme activities and synthesis of macromolecules [6], reduction of antioxidants $[3,6]$, cessation of cell growth, reduction of viability [7] and inhibition of cell proliferation and differentiation, gene mutation, delay of cell cycle, induction of cell apoptosis and necrosis $[3,6]$.

Acrylic resin cytotoxicity is associated, most times, with the presence of residual monomers in the polymerization process. The monomers change cell morphology and function which can reduce their viability.

Since acrylic resins are widely used in dental practice, an adequate biocompatibility is essential. Considering that the majority of studies reported acrylic resin toxicity responses, further studies with different assessment methods are necessary for the development of biocompatible materials.

\section{Final Considerations}

There are different methods to evaluate acrylic resin cytotoxicity, with the MTT method being the most common test. In general, there is not a non-cytotoxic acrylic resin available on the dental market. Regarding the polymerization method, the auto-polymerized resin is more cytotoxic than heat-polymerized resin. The cytotoxic effect is dose dependent and depends on the amount of residual monomers. Residual monomers are responsible for inflammatory reactions of tissues in contact with the acrylic resin.

\section{References}

[1] Acosta-Torres LS, Mendieta I, Nuñez-Anita RE, Cajero-Juárez M, Castaño VM: Cytocompatible antifungal acrylic resin containing silver nanoparticles for dentures. Int J Nanomedicine 2012, 7, 4777-4786.

[2] Ata SO, Yavuzyilmaz H: In vitro comparison of the cytotoxicity of acetal resin, heat-polymerized resin, and autopolymerized resin as denture base materials. J Biomed Mater Res B Appl Biomater 2009, 91, 905-909.

[3] Kojima N, Yamada M, Paranjpe A, Tsukimura N, Kubo K, Jewett A, Ogawa T: Restored viability and function of dental pulp cells on poly-methylmethacrylate (PMMA)-based dental resin supplemented with N-acetyl cysteine (NAC). Dent Mater 2008, 24, 1686-1693.

[4] Hattori N, Suzuki T, Jinno H, Okeya A, Ishikawa A, Kondo C, Hayashi T, Ito M, Kanamori T, Kawai T, Noguchi T: Methyl methacrylate activates the Gsta1 Promoter. J Dent Res 2008, 87, 1117-1121.

[5] Tay LY, Herrera DR, Quishida CC, Carlos IZ, Jorge JH: Effect of water storage and heat treatment on the cytotoxicity of soft liners. Gerodontology 2012, 29, 275-280.

[6] Att W, Yamada M, Kojima N, Ogawa T: N-Acetyl cysteine prevents suppression of oral fibroblast function on poly(methylmethacrylate) resin. Acta Biomater 2009, 5, 391-398.

[7] Yamada M, Kojima N, Att W, Hori N, Suzuki T, Ogawa T: N-Acetyl cysteine restores viability and function of rat odontoblast-like cells impaired by polymethylmethacrylate dental resin extract. Redox Rep 2009, 14, 13-22.

[8] Bural C, Aktaş E, Deniz G, Ünlüçerçi Y, Bayraktar G: Effect of leaching residual methyl methacrylate concentrations on in vitro cytotoxicity of heat polymerized denture base acrylic resin processed with different polymerization cycles. J Appl Oral Sci 2011, 19, 306-312.

[9] Chaves CA, Machado AL, Vergani CE, de Souza RF, Giampaolo ET: Cytotoxicity of denture base and hard chairside reline materials: a systematic review. J Prosthet Dent 2012, 107, 114-127.

[10] Ebrahimi Saravi M, Vojdani M, Bahrani F: Evaluation of cellular toxicity of three denture base acrylic resins. J Dent (Tehran) 2012, 9, 180-188.

[11] Bural C, Aktaş E, Deniz G, Ünlüçerçi Y, Kizılcan N, Bayraktar G: Effect of post-polymerization heat-treatments on degree of conversion, leaching residual MMA and in vitro cytotoxicity of autopolymerizing acrylic repair resin. Dent Mater 2011b, 27, 1135-1143.

[12] Regis RR, Della Vecchia MP, Pizzolitto AC, Compagnoni MA, Souza PP, de Souza RF: Antimicrobial properties and cytotoxicity of an antimicrobial monomer for application in prosthodontics. J Prosthodont 2012, 21, $283-290$.

[13] Zheng J, Su Q, Wang C, Cheng G, Zhu R, Shi J, Yao K: Synthesis and biological evaluation of PMMA/MMT nanocomposite as denture base material. J Mater Sci Mater Med 2011, 22, 1063-1071.

[14] Trubiani O, Toniato E, Di Iorio D, Diomede F, Merciaro I, D’ Arcangelo C, Caputi S: Morphological analysis and interleukin release in human gingival fibroblasts seeded on different denture base acrylic resins. Int J Immunopathol Pharmacol 2012, 25, 637-643.

[15] Kanie T, Tomita K, Tokuda M, Arikawa H, Fujii K, Ban S: Mechanical properties and cytotoxicity of experimental soft lining materials based on urethane acrylate oligomers. Dent Mater J 2009, 28, 501-506. 
[16] Segerström S, Sandborgh-Englung G, Ruyter EI: Biological and physicochemical properties of carbon-graphite fibre-reinforced polymers intended for implant suprastructures. Eur J Oral Sci 2011, 119, 246-252.

[17] Dawlee S, Jayakrishnan A, Jayabalan M: Studies on novel radiopaque methyl methacrylate: glycidyl methacrylate based polymer for biomedical applications. J Mater Sci Mater Med 2009, 20, 243-250.

[18] Cochis A, Fracchia L, Martinotti MG, Rimondini L: Biosurfactants prevent in vitro Candida albicans biofilm formation on resins and silicon materials for prosthetic devices. Oral Surg Oral Med Oral Pathol Oral Radiol 2012, $113,755-761$.

[19] Borak J, Fields C, Andrews LS, Pemberton MA: Methyl methacrylate and respiratory sensitization: a critical review. Crit Rev Toxicol 2011, 41, 230-268.

\section{Address for correspondence:}

Marcelo C. Goiato

Rua José Bonifácio, 1193, Vila Mendonça

Araçatuba

São Paulo

Brazil 16015-050

Tel.: +551836363287

E-mail: goiato@foa.unesp.br

Conflict of interest: None declared

Received: 25.09.2014

Revised: 14.10.2014

Accepted: 13.11.2014 